\title{
Cavity optomagnonics with magnetic textures: Coupling a magnetic vortex to light
}

\author{
Jasmin Graf, ${ }^{1}$ Hannes Pfeifer, ${ }^{1}$ Florian Marquardt, ${ }^{1,2}$ and Silvia Viola Kusminskiy ${ }^{1}$ \\ ${ }^{1}$ Max Planck Institute for the Science of Light, Staudtstraße 2, 91058 Erlangen, Germany \\ ${ }^{2}$ Institute for Theoretical Physics, University Erlangen-Nürnberg, Staudtstraße 2, 91058 Erlangen, Germany
}

(Received 17 July 2018; revised manuscript received 7 November 2018; published 5 December 2018)

\begin{abstract}
Optomagnonic systems, where light couples coherently to collective excitations in magnetically ordered solids, are currently of high interest due to their potential for quantum information processing platforms at the nanoscale. Efforts so far, both at the experimental and theoretical level, have focused on systems with a homogeneous magnetic background. A unique feature in optomagnonics is, however, the possibility of coupling light to spin excitations on top of magnetic textures. We propose a cavity-optomagnonic system with a nonhomogeneous magnetic ground state, namely, a vortex in a magnetic microdisk. In particular, we study the coupling between optical whispering gallery modes to magnon modes localized at the vortex. We show that the optomagnonic coupling has a rich spatial structure and that it can be tuned by an externally applied magnetic field. Our results predict cooperativities at maximum photon density of the order of $\mathcal{C} \approx 10^{-2}$ by proper engineering of these structures.
\end{abstract}

DOI: 10.1103/PhysRevB.98.241406

Introduction. Optomagnonics is an exciting new field where light couples coherently to elementary excitations in magnetically ordered systems. The origin of this photonmagnon interaction is the Faraday effect, where the magnetization in the sample causes the light's polarization plane to rotate. Conversely, the light exerts a small effective magnetic field on the material's magnetic moments. Shaping the host material into an optical cavity enhances the effective coupling according to the increased number of trapped photons.

Recent seminal experiments have demonstrated this coupling [1-3]. In these, an yttrium iron garnet (YIG) sphere serves as the host of the magnetic excitations and, via whispering gallery modes (WGMs), as the optical cavity. The optomagnonic coupling manifests itself in transmission sidebands at the magnon frequency. So far, these experiments have probed mostly the homogeneous magnetic mode (Kittel mode) where all spins rotate in phase [4]. Very recently, optomagnonic coupling to other magnetostatic modes $[5,6]$ has been demonstrated, albeit still on top of a homogeneous background $[7,8]$.

The Kittel mode, although it is the simplest one to probe and externally tune, is a bulk mode and has a suboptimal overlap with the optical WGMs living near the surface. Another caveat is the state of the art in terms of sample size, which is currently submillimetric. This results in modest values for the optomagnonic coupling and motivates the quest for smaller, micron-sized magnetic samples, as well as for engineering the coupling between magnetic and optical modes. Increasing the currently observed values of optomagnonic coupling is an urgent prerequisite for moving on to promising applications such as magnon cooling, coherent state transfer, or efficient wavelength converters [9-24].

In microscale magnetic samples, the competition between the short-range exchange interaction and the boundarysensitive demagnetization fields can lead to magnetic textures, where the magnetic ground state is not homogeneous $[25,26]$.
A well-studied case is that of a thin microdisk, where the magnetization swirls in the plane of the disk and forms a magnetic vortex in the center [27,28] [see Fig. 1(a)]. In the vortex core, the spins point out of plane.

Magnetic vortices carry two degrees of freedom: How the magnetization curls (clockwise or anticlockwise) defines the chirality $\mathcal{C}= \pm 1$, while its pointing up or down at the center of the vortex defines the polarity $\mathcal{P}= \pm 1$. These are robust topological properties and make vortices interesting for information processing [26]. Moreover, the position of the vortex can be controlled by an external magnetic field, making this system highly tunable [Fig. 1(c)].

While optomagnonic systems present analogies to optomechanics [16,29] (where light couples to phonons [30]), the possibility of coupling light to magnetic textures is unique to optomagnonics. In this Rapid Communication we study the optomagnonic coupling in the presence of an inhomogeneous magnetic background in a microdisk geometry (note that this differs from Refs. [6-8], where the underlying magnetic
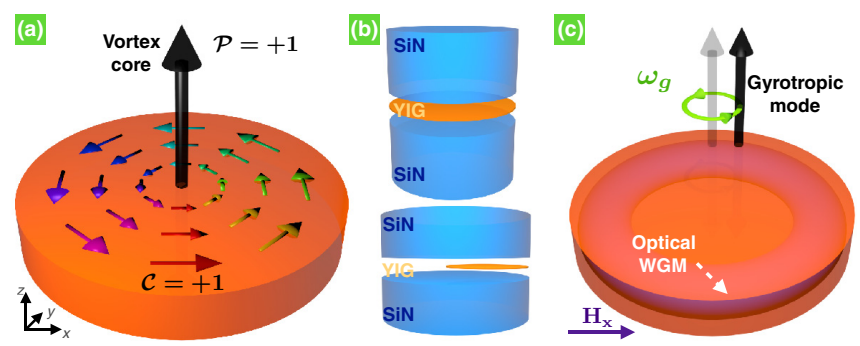

FIG. 1. (a) In a magnetic microdisk, the ordered state is a vortex with chirality $\mathcal{C}= \pm 1$ and polarity $\mathcal{P}= \pm 1$. Envisioned setups: (b) A thin YIG disk embedded in an optical cavity of choice. (c) A thicker YIG microdisk can support both optical WGM and magnon modes. An external in-plane magnetic field displaces the vortex core in a direction perpendicular to the field. 
ground state is uniform). This is a relevant case to study since: (i) YIG disks at the microscale have been experimentally realized and the presence of magnetic vortices demonstrated [31-33], (ii) a disk supports optical WGMs while reducing the magnetic volume with respect to a sphere, which could lead to larger optomagnonic couplings, and (iii) the spin excitations in the presence of the vortex are qualitatively different from those on top of a homogeneous magnetization.

We combine analytical methods with micromagnetic and finite-element simulations to derive the spatial dependence and the strength of the optomagnonic coupling. We study two qualitatively different regimes that can be accessed by nanostructure patterning: a very thin micromagnetic disk embedded in an optical cavity, and a thicker microdisk that also serves as the optical cavity [Figs. 1(b) and 1(c)]. We demonstrate our method for the coupling between magnon modes localized at the magnetic vortex and the optical WGMs, and predict high values for the optomagnonic coupling and the cooperativity, an important figure of merit in these systems.

Optomagnonic coupling for magnetic textures. In a Faraday active material, the electromagnetic energy is modified by the coupling between the electric field and the magnetization [34],

$$
H_{\mathrm{MO}}=-i \frac{\theta_{\mathrm{F}} \lambda_{n}}{2 \pi} \frac{\varepsilon_{0} \varepsilon}{2} \int d \mathbf{r} \mathbf{m}(\mathbf{r}, t) \cdot\left[\mathbf{E}^{*}(\mathbf{r}, t) \times \mathbf{E}(\mathbf{r}, t)\right],
$$

where $\mathbf{m}(\mathbf{r}, t)$ is the local magnetization in units of the saturation magnetization $M_{\mathrm{s}}$, and we used the complex representation of the electric field, $\left(\mathbf{E}^{*}+\mathbf{E}\right) / 2$. The prefactor $\theta_{\mathrm{F}} \lambda_{n} / 2 \pi\left(\sim 4 \times 10^{-5}\right.$ in $\left.\mathrm{YIG}\right)$ gives the Faraday rotation $\theta_{\mathrm{F}}$ per wavelength $\lambda_{n}$ in the material, $\varepsilon\left(\varepsilon_{0}\right)$ is the relative (vacuum) permittivity, and $n=\sqrt{\varepsilon / \varepsilon_{0}}$ the refractive index. Equation (1) couples the spin density in the magnetic material with the optical spin density (OSD), which represents the spin angular momentum density carried by the light field. Quantizing Eq. (1) leads to the optomagnonic Hamiltonian [29]. The coupling is parametric, coupling one local spin operator to two photon operators.

We consider the coupling of the optical fields to spinwave excitations on top of a nonuniform static ground state $\mathbf{m}_{0}(\mathbf{r}), \delta \mathbf{m}(\mathbf{r}, t)=\mathbf{m}(\mathbf{r}, t)-\mathbf{m}_{0}(\mathbf{r})$. For small deviations $|\delta \mathbf{m}| \ll 1$ we can express these in terms of harmonic oscillators (magnon modes). Quantizing $\delta \mathbf{m}(\mathbf{r}, t) \rightarrow$ $\frac{1}{2} \sum_{\gamma}\left[\delta \mathbf{m}_{\gamma}(\mathbf{r}) \hat{b}_{\gamma} e^{-i \omega_{\gamma} t}+\delta \mathbf{m}_{\gamma}^{*}(\mathbf{r}) \hat{b}_{\gamma}^{\dagger} e^{i \omega_{\gamma} t}\right]$ and $\mathbf{E}^{(*)}(\mathbf{r}, t) \rightarrow$ $\sum_{\beta} \mathbf{E}_{\beta}^{(*)}(\mathbf{r}) \hat{a}_{\beta}^{(\dagger)} e^{-(+) i \omega_{\beta} t}$, from Eq. (1) we obtain the coupling Hamiltonian $\hat{H}_{\mathrm{MO}}=\sum_{\alpha \beta \gamma} G_{\alpha \beta \gamma} \hat{a}_{\alpha}^{\dagger} \hat{a}_{\beta} \hat{b}_{\gamma}+$ H.c., where $G_{\alpha \beta \gamma}=\int d \mathbf{r} G_{\alpha \beta \gamma}(\mathbf{r})$ and

$$
G_{\alpha \beta \gamma}(\mathbf{r})=-i \frac{\theta_{\mathrm{F}} \lambda_{n}}{4 \pi} \frac{\varepsilon_{0} \varepsilon}{2} \delta \mathbf{m}_{\gamma}(\mathbf{r}) \cdot\left[\mathbf{E}_{\alpha}^{*}(\mathbf{r}) \times \mathbf{E}_{\beta}(\mathbf{r})\right]
$$

is the local optomagnonic coupling. The Greek subindices indicate the respective magnon and photon modes which are coupled. We use Eq. (2) to evaluate the coupling between optical WGMs and magnon modes in a YIG microdisk with a magnetic vortex, focusing on magnonic modes localized at the vortex. We study two cases: (i) a thin disk where the problem is essentially two dimensional (2D), and (ii) a thicker disk,
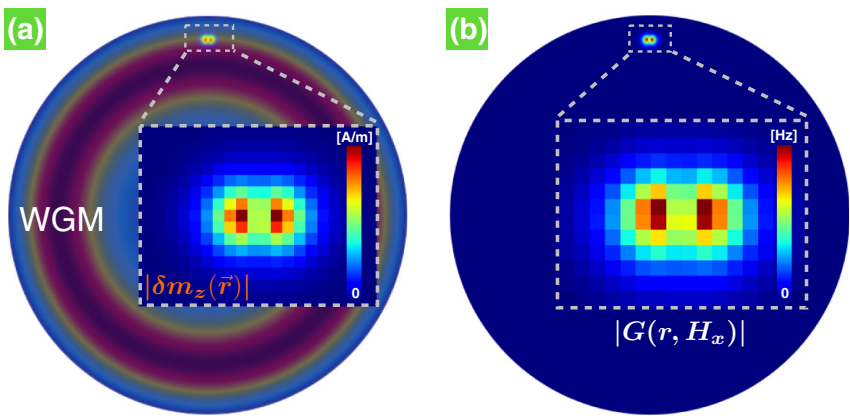

FIG. 2. Optomagnonic coupling to vortex motion in a thin disk. (a) Optical WGM and gyrotropic mode (inset) for a displaced vortex. (b) Spatial profile of the optomagnonic coupling. ( $R=1 \mu \mathrm{m}, h=$ $20 \mathrm{~nm}, \omega_{\text {opt }} / 2 \pi=217 \mathrm{THz}, \kappa_{\text {opt }}=1.51 \mathrm{THz}, \omega_{g} / 2 \pi=36 \mathrm{MHz}$, $H_{x}=3.3 \mathrm{mT}$.)

where the $z$ dependence of the problem is nontrivial. The thin disk allows us to compare with analytical approximate results, validating our numerical results.

Thin disk. We consider a magnetic microdisk of thickness $h$ and radius $R$. The characteristic magnetic length scale is the exchange length $l_{\mathrm{ex}}$ (for YIG: $l_{\mathrm{ex}} \sim 10 \mathrm{~nm}$ ) [35]. A vortex is the stable magnetic texture for $h \gtrsim l_{\mathrm{ex}}$ and $R \gg$ $l_{\text {ex }}$ [28]. The lowest excitation mode consists of the vortex's center-of-mass rotating around an axis perpendicular to the disk's plane [36,37] [see Fig. 1(c)]. The frequency $\omega_{g}$ of this gyrotropic mode can be approximated by $\omega_{g} / 2 \pi \approx$ $\mu_{0} \gamma M_{\mathrm{s}} h /(4 \pi R)$ with $\gamma$ the gyromagnetic ratio [28] (for YIG $\left.\omega_{g} / 2 \pi \approx 1 \mathrm{GHz} h / R\right)$. The excitation is localized at the vortex core, decaying linearly with distance (see Supplemental Material (SM) A [38]). The disk also supports optical WGMs. The approximate 2D analytical solution for these is well known (see SM C [38]). The WGMs can be classified into transverse magnetic (TM) and transverse electric (TE) modes, for electric field perpendicular to and in the plane of the disk, respectively [39]. Within this approximation we have two possibilities for finite coupling to the gyrotropic mode: processes involving both TE and TM modes, and those involving only TE modes. For processes involving both TE and TM modes, $\mathbf{E}_{\alpha}^{\mathrm{TE} *} \times \mathbf{E}_{\beta}^{\mathrm{TM}}$ lies in the $x y$ plane and therefore can couple to the in-plane component of the gyrotropic mode, which is finite both inside and outside of the vortex core. Processes involving instead only TE modes couple exclusively to the out-of-plane component of the gyrotropic mode, which is finite only inside the vortex core (SM A [38]). For a YIG microdisk, the free spectral range $\Delta f_{\mathrm{FSR}} \approx 0.1 \mathrm{THz}$, which is much larger than the typical gyrotropic frequencies. Therefore, magnon scattering between two energetically distinct optical modes would be allowed either in the sideband unresolved case, or possibly with carefully selected modes of other radial optical quantum numbers. Moreover, using an external magnetic field for frequency matching can be difficult in these structures, since it would alter the static magnetic texture and consequently the modes. In the following we discuss the case of scattering with one TE mode, which is free from these considerations. This is analogous to single-mode optomechanics [30] or optomagnonics [29], where the system 
is driven by a laser whose detuning from the optical mode can be made to match the magnon frequency.

Coupling to the gyrotropic mode is only possible if there is an overlap with the WGM. Applying a magnetic field $H_{x}$ along $x$ displaces the vortex up (down) along $y$ for counterclockwise (clockwise) chirality, as the spins try to align with the field. This provides a knob to control the optomagnonic coupling, as we show in the following. We first note, however, that a thin YIG microdisk such that $h \gtrsim l_{\mathrm{ex}} \approx 10 \mathrm{~nm}$ is a bad optical cavity. To better confine the optical modes, we consider a structure as shown in Fig. 1(b), such that the YIG disk is placed between two dielectric, nonmagnetic disks with the same radius and height comparable to $\lambda_{n}$. We chose $\mathrm{Si}_{3} \mathrm{~N}_{4}$ (refractive index $n_{\mathrm{Si}_{3} \mathrm{~N}_{4}} \approx 2$ ) in order to create an almost continuous material for the WGM resonator. Hence the WGMs live in the whole structure, whereas the magnon modes are confined to the thin YIG disk. The gyrotropic mode can overlap with the WGMS for a displaced vortex [see Fig. 2(a)]. We continue to call this mode "gyrotropic" since it evolves continuously from the gyrotropic mode at $H_{x}=0$. Whereas its frequency has a light dependence on $H_{x}$, the mode itself is distorted as the rim of the disk is approached. This reflects the deformation of the vortex core into a $\mathrm{C}$-shaped domain wall due to the stronger influence of the demagnetization fields at the nearest boundary (see SM D [38] and Refs. [40,41]).

Figure 2 shows an example of the spatial dependence of the optomagnonic coupling $\left|G\left(\mathbf{r}, H_{x}\right)\right|$ for the gyrotropic mode and a WGM. The coupling, given throughout this work as per photon and per magnon, was obtained by combining $\mathrm{MuMax}^{3}$ [42] micromagnetic simulations with finiteelement simulations for the optical WGM via Eq. (2). Details on the simulations and the normalization procedure are presented in SM F, G, and $\mathrm{H}$ [38]. The total coupling is obtained by integrating over the whole volume. The integration volume is however bounded by the magnon mode volume $V_{\text {mag }}$ since it is smaller than the optical mode $V_{\text {opt }}$. A quick estimate of the maximum coupling is $\left|G_{\max }\right| \approx$ $\sqrt{2 g \mu_{B} /\left(M_{\mathrm{s}} V_{\mathrm{mag}}\right)}\left(\theta_{\mathrm{F}} \lambda_{n} / 2 \pi\right)\left(V_{\mathrm{mag}} / V_{\mathrm{opt}}\right) \hbar \omega_{\mathrm{opt}}(g$ is the $g-$ factor, $\mu_{B}$ is the Bohr magneton), showing a suppression of the coupling by a factor $\sqrt{V_{\mathrm{mag}}} / V_{\mathrm{opt}}$. For the thin disk considered here we find $\left|G_{\max }\right| \approx 30 \mathrm{~Hz}$, in agreement with the modest maximum value of $|G| \approx 10 \mathrm{~Hz}$ obtained numerically (see Fig. 3). Interestingly, this maximum value is not obtained at the maximum of the OSD, but at points of its maximum slope (as a function of vortex position). This can be understood by noting the antisymmetry under inversion of $\delta m_{z}$ for the gyrotropic mode, which leads to a cancellation when integrated weighted by an isotropic factor. This cancellation is lifted most effectively when the vortex is located at highly anisotropic points of the OSD. Figure 3 shows $|G|$ as a function of applied magnetic field, together with the profile for the OSD. This shows clearly that the magnon mode couples effectively to the gradient of the OSD. The value of $|G|$ is therefore completely tunable by an external magnetic field, in contrast to the usual case of magnonic modes on a homogeneous background.

Using the "rigid vortex" model $[28,43]$ for the magnetics, the optomagnonic coupling for the gyrotropic mode can be obtained analytically (SM A, B, and D [38]). Using that the vortex core radius $b$ is small, the first nonzero contribution to

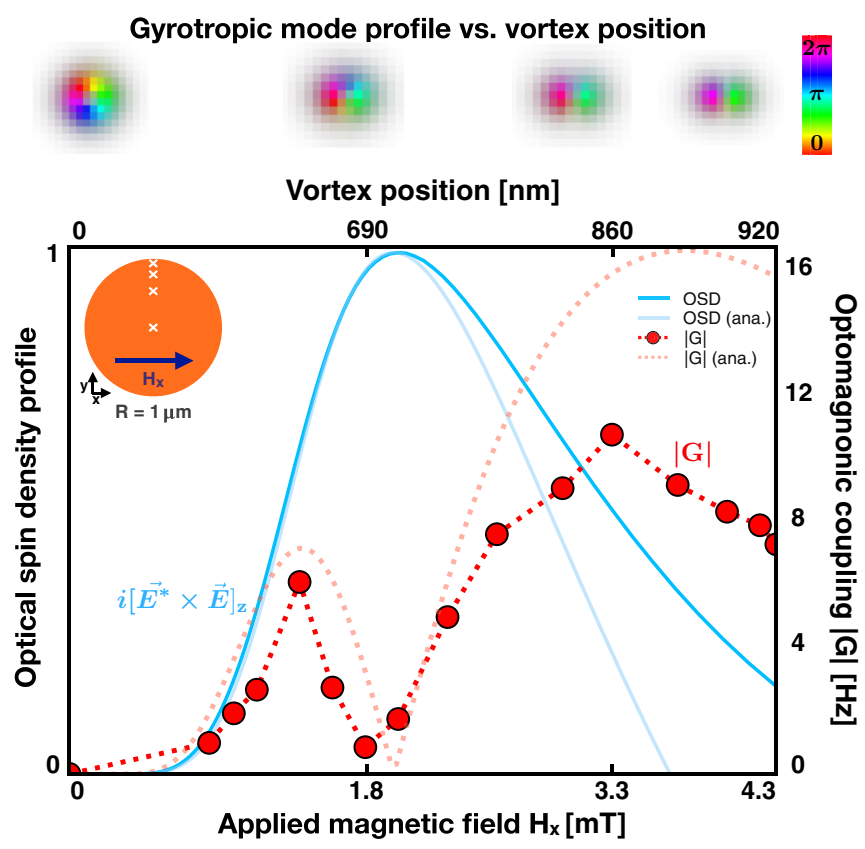

FIG. 3. Optomagnonic coupling (red dots: numerical; light-red dotted line: analytical) as a function of magnetic field $H_{x}$ (bottom axis, linear scale) or vortex position (top axis) for the thin disk, and OSD (blue solid line: numerical; light blue solid line: analytical) at the position of the vortex, normalized to the maximum OSD. Note the nonlinear dependence of the position of the vortex on $H_{x}$ (see SM E [38]). The vortex position is shown schematically in the inset. Above the graph, the complex-valued mode function $\delta m_{z}(\mathbf{r})$ shows the distortion of the gyrotropic mode as the vortex moves close to the rim. The analytical model does not consider this nor the decay of the optical field, which accounts for the deviations near the rim of the disk. ( $R=1 \mu \mathrm{m}, h=20 \mathrm{~nm}, \omega_{\mathrm{opt}} / 2 \pi=217 \mathrm{THz}$, $\kappa_{\text {opt }}=1.51 \mathrm{THz}$.)

the coupling in a Taylor expansion is

$$
\left|G_{m}(s)\right| \approx 10^{-2} \theta_{\mathrm{F}} \lambda_{n} \sqrt{\frac{g \mu_{B} h}{M_{\mathrm{s}}}} b^{2} \varepsilon_{0} \varepsilon\left|\partial_{s}\left[\left(\mathbf{E}_{m}^{\mathrm{TE} *} \times \mathbf{E}_{m}^{\mathrm{TE}}\right)_{z}\right]\right|,
$$

with $s$ the vortex position and $m$ the WGM label. This confirms the coupling to the gradient of the OSD. This simplified analytical model is in good agreement with the simulations (see Fig. 3).

Thick disk. The magnetic texture can be considered independent of height when $h$ is only a few $l_{\text {ex }}$. Increasing the height of the disk leads to more complex magnetic textures and the appearance of magnon flexural modes along the $z$ direction [44], which can hybridize with in-plane modes [45]. Although this effect is already present for $H_{x}=0$, it is even more striking for a finite external field. We discuss this regime in the following.

We consider a "thick" microdisk such that $h \gg l_{\mathrm{ex}}$ in an applied external field $H_{x}$. In this case the vortex "snakes" from the top to the bottom of the disk [see Figs. 4(a)-4(c)]. This results in highly complex magnon modes, which we obtain by micromagnetic simulations. The spatial structure for the first excited modes is shown in Fig. 4(g). We interpret these 

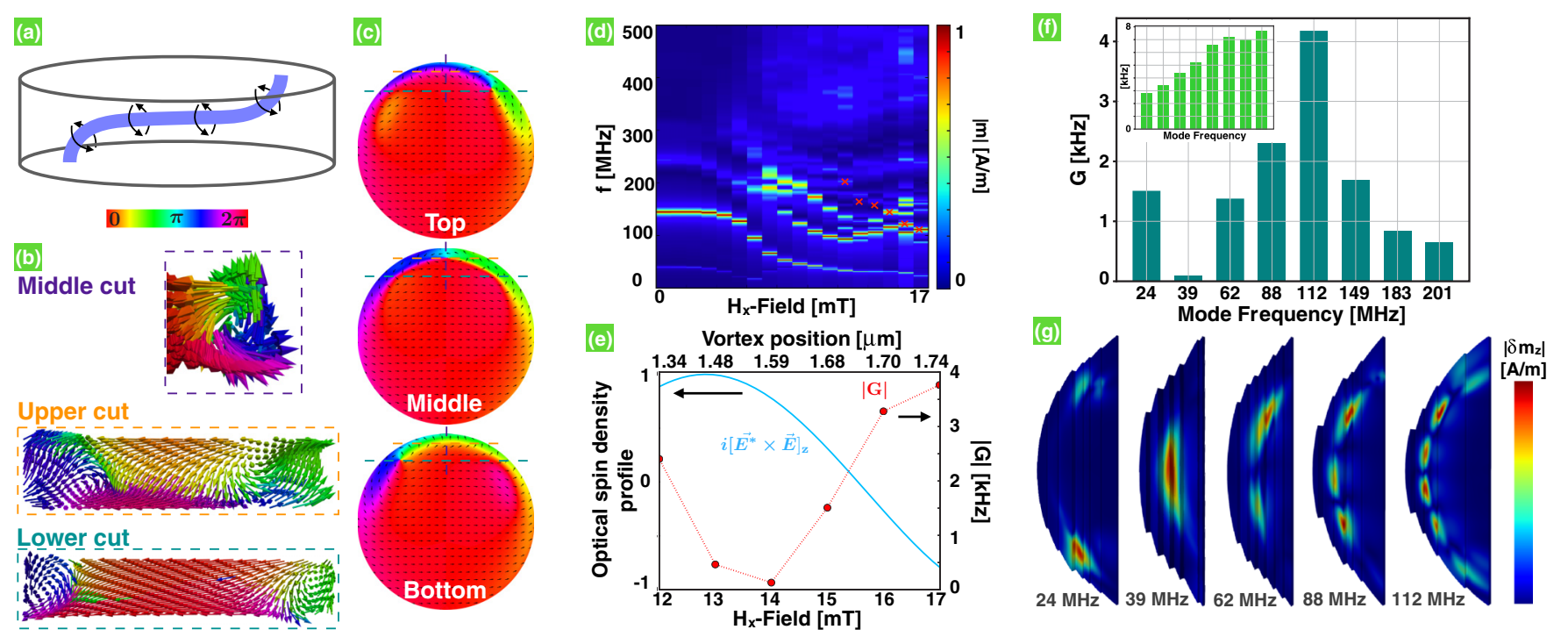

FIG. 4. Magnetic background, localized magnon modes, and optomagnonic coupling for a thick disk. (a) Sketch of the magnetic vortex. (b) and (c) Sectional cuts showing the 3D structure of the vortex. (d) Magnon spectrum vs magnetic field. The red crosses indicate the magnon mode considered in plot (e). (e) Coupling as a function of magnetic field (red dots), and OSD (blue solid line) at the vortex position. (f) Coupling as a function of magnon mode frequency. The inset shows $\int_{V} d^{3} r|G(\mathbf{r})|$ for comparison. (g) Profile of the first five excited magnon modes. For (b), (c), (f), and (g), $H_{x}=17 \mathrm{mT}$. All couplings for a TE-WGM of $\omega_{\text {opt }} / 2 \pi=184 \mathrm{THz}, \kappa_{\text {opt }}=0.03 \mathrm{THz} .(R=2 \mu \mathrm{m}, h=500 \mathrm{~nm}$. $)$

as flexural modes of the vortex core, possibly hybridized with the gyrotropic mode. The optomagnonic coupling for these modes at a fixed $H_{x}$ is presented in Fig. 4(f). We observe that (i) we obtain values for the coupling in the $\mathrm{kHz}$ range, and (ii) the value of the coupling has a nonmonotonic dependence on the mode number, due to cancellation effects, as can be seen when compared with the integrated absolute value of the coupling. This system shows also tunability by an external magnetic field, and the coupling is governed by the gradient of the OSD (see Fig. 4). Taking the Gilbert damping coefficient for YIG $\alpha \approx 10^{-5}$, we obtain single-photon cooperativities up to $\mathcal{C}_{0}=4 G^{2} /(\kappa \alpha \omega) \sim 10^{-7}$, where $\kappa \sim 0.1 \mathrm{THz}$ (from COMSOL) and $\omega$ frequency of the respective magnon mode. For a maximum allowed photon density of $10^{5} \mu \mathrm{m}^{-3}, \mathcal{C}=$ $n_{\mathrm{ph}} \mathcal{C}_{0} \sim 10^{-2}$, a five orders of magnitude improvement with respect to the current state of the art.

Conclusion. We developed a numerical method based in micromagnetics and finite-element simulations for cavity optomagnonics with magnetic textures. We studied a microdisk where the magnetic static background is a vortex. The system presents two qualitatively distinct regimes. For thin disks the problem allows for an approximate analytical treatment, which we use to benchmark our results. For this case, we propose a heterostructure where the optical cavity surrounds the microdisk for better confinement of the optical modes. A simpler structure from the experimental point of view could be instead an optical cavity on top of the microdisk, where the coupling is evanescent. This could provide the freedom of designing optical modes independently of the magnetic structure. For thick disks, the microdisk serves also as the optical cavity. This system presents a rich magnetic structure, and large values of optomagnonic coupling and cooperativities are in principle achievable. Coupling to other spin-wave modes in microdisks, of the WGM kind [46], could boost these values even further. The predicted values imply a significant improvement with respect to the state of the art, and are attainable within current technology. Our results pave the way for optomagnonics with magnetic textures [47,48], including optically induced nonlinear vortex dynamics (e.g., self-oscillations of the gyrotropic mode), optically mediated synchronization in vortex arrays, and exotic quantum states entangling vortex and optical degrees of freedom. Finally, our results indicate the potential of these systems for cavityenhanced Brillouin scattering microscopy to study vortices or other magnetic structures.

Acknowledgments. We thank A. Aiello for discussions and K. Usami for useful comments on the manuscript. F.M. acknowledges support through the European FET proactive network "Hybrid Optomechanical Technologies." S.V.K. acknowledges support from the Max Planck Gesellschaft through an Independent Max Planck Research Group.
[1] J. A. Haigh, A. Nunnenkamp, A. J. Ramsay, and A. J. Ferguson, Phys. Rev. Lett. 117, 133602 (2016).

[2] A. Osada, R. Hisatomi, A. Noguchi, Y. Tabuchi, R. Yamazaki, K. Usami, M. Sadgrove, R. Yalla, M. Nomura, and Y. Nakamura, Phys. Rev. Lett. 116, 223601 (2016).
[3] X. Zhang, N. Zhu, C.-L. Zou, and H. X. Tang, Phys. Rev. Lett. 117, 123605 (2016).

[4] C. Kittel, Phys. Rev. 73, 155 (1948).

[5] S. Sharma, Y. M. Blanter, and G. E. W. Bauer, Phys. Rev. B 96, 094412 (2017). 
[6] A. Osada, A. Gloppe, Y. Nakamura, and K. Usami, New J. Phys. 20, 103018 (2018).

[7] A. Osada, A. Gloppe, R. Hisatomi, A. Noguchi, R. Yamazaki, M. Nomura, Y. Nakamura, and K. Usami, Phys. Rev. Lett. 120, 133602 (2018).

[8] J. A. Haigh, N. J. Lambert, S. Sharma, Y. M. Blanter, G. E. W. Bauer, and A. J. Ramsay, Phys. Rev. B 97, 214423 (2018).

[9] O. O. Soykal and M. E. Flatté, Phys. Rev. Lett. 104, 077202 (2010).

[10] H. Huebl, C. W. Zollitsch, J. Lotze, F. Hocke, M. Greifenstein, A. Marx, R. Gross, and S. T. B. Goennenwein, Phys. Rev. Lett. 111, 127003 (2013).

[11] X. Zhang, C.-L. Zou, L. Jiang, and H. X. Tang, Phys. Rev. Lett. 113, 156401 (2014).

[12] Y. Tabuchi, S. Ishino, A. Noguchi, T. Ishikawa, R. Yamazaki, K. Usami, and Y. Nakamura, Science 349, 405 (2015).

[13] J. A. Haigh, N. J. Lambert, A. C. Doherty, and A. J. Ferguson, Phys. Rev. B 91, 104410 (2015).

[14] X. Zhang, C. Zou, L. Jiang, and H. X. Tang, J. Appl. Phys. 119, 023905 (2016).

[15] X. Zhang, C.-L. Zou, L. Jiang, and H. X. Tang, Sci. Adv. 2, e1501286 (2016).

[16] T. Liu, X. Zhang, H. X. Tang, and M. E. Flatté, Phys. Rev. B 94, 060405 (2016).

[17] Y. Tabuchi, S. Ishino, A. Noguchi, T. Ishikawa, R. Yamazaki, K. Usami, and Y. Nakamura, C. R. Phys. 17, 729 (2016).

[18] D. Lachance-Quirion, Y. Tabuchi, S. Ishino, A. Noguchi, T. Ishikawa, R. Yamazaki, and Y. Nakamura, Sci. Adv. 3, e1603150 (2017).

[19] J. Bourhill, N. Kostylev, M. Goryachev, D. L. Creedon, and M. E. Tobar, Phys. Rev. B 93, 144420 (2016).

[20] H. Maier-Flaig, M. Harder, S. Klingler, Z. Qiu, E. Saitoh, M. Weiler, S. Geprägs, R. Gross, S. T. B. Goennenwein, and H. Huebl, Appl. Phys. Lett. 110, 132401 (2017).

[21] M. Harder, L. Bai, P. Hyde, and C.-M. Hu, Phys. Rev. B 95, 214411 (2017).

[22] P. A. Pantazopoulos, N. Stefanou, E. Almpanis, and N. Papanikolaou, Phys. Rev. B 96, 104425 (2017).

[23] R. G. E. Morris, A. F. van Loo, S. Kosen, and A. D. Karenowska, Sci. Rep. 7, 11511 (2017).

[24] S. Sharma, Y. M. Blanter, and G. E. W. Bauer, Phys. Rev. Lett. 121, 087205 (2018).

[25] A. Hubert and R. Schäfer, Magnetic Domains - The Analysis of Magnetic Microstructures (Springer, Berlin, 1998).

[26] A. P. Guimarães, Principles of Nanomagnetism (Springer, Berlin, 2017).

[27] T. Shinjo, T. Okuno, R. Hassdorf, K. Shigeto, and T. Ono, Science 289, 930 (2000).

[28] K. Y. Guslienko, J. Nanosci. Nanotechnol. 8, 2745 (2008).

[29] S. Viola Kusminskiy, H. X. Tang, and F. Marquardt, Phys. Rev. A 94, 033821 (2016).

[30] M. Aspelmeyer, T. J. Kippenberg, and F. Marquardt, Rev. Mod. Phys. 86, 1391 (2014).

[31] J. E. Losby, F. F. Sani, D. T. Grandmont, Z. Diao, M. Belov, J. A. J. Burgess, S. R. Compton, W. K. Hiebert, D. Vick, K. Mohammad, E. Salimi, G. E. Bridges, D. J. Thomson, and M. R. Freeman, Science 350, 798 (2015).
[32] M. Collet, X. de Milly, O. d'Allivy Kelly, V. V. Naletov, R. Bernard, P. Bortolotti, J. B. Youssef, V. E. Demidov, S. O. Demokritov, J. L. Prieto, M. Muñoz, V. Cros, A. Anane, G. de Loubens, and O. Klein, Nat. Commun. 7, 10377 (2016).

[33] N. Zhu, H. Chang, A. Franson, T. Liu, X. Zhang, E. JohnstonHalperin, M. Wu, and H. X. Tang, Appl. Phys. Lett. 110, 252401 (2017).

[34] L. D. Landau, L. P. Pitaevskii, and E. M. Lifshitz, Electrodynamics of Continuous Media, 2nd ed., Course of Theoretical Physics (Pergamon, Oxford, U.K., 1984), Vol. 8.

[35] Another important scale for domain formation is the anisotropy constant $K$, which we assume to be small.

[36] A. A. Thiele, Phys. Rev. Lett. 30, 230 (1973).

[37] K. Y. Guslienko, B. A. Ivanov, V. Novosad, Y. Otani, H. Shima, and K. Fukamichi, J. Appl. Phys. 91, 8037 (2002).

[38] See Supplemental Material at http://link.aps.org/supplemental/ 10.1103/PhysRevB.98.241406 for (A) a detailed derivation of the mathematical expressions for the gyrotropic mode on top of the magnetic texture of a vortex, which includes Refs. [28,43]. (B) for details on the analytical normalization of the optomagnonic coupling, which includes Ref. [49]; (C) for a detailed derivation of the mathematical expressions for the whispering gallery modes hosted by a cylindrical cavity, which includes Ref. [50]; (D) for a detailed derivation of the optomagnonic coupling and its proportionality to the spin density of light; (E) for details on how the vortex position can be translated into a external magnetic field, which includes Ref. [51]; (F) for details on the optical simulations; (G) for details on the micromagnetic simulations, which includes Refs. [31,42]; and $(\mathrm{H})$ for details on the numerical normalization of the optomagnonic coupling.

[39] Note that in some works the opposite convention is used; see, e.g., Ref. [2].

[40] K. Rivkin, W. Xu, L. E. D. Long, V. V. Metlushko, B. Ilic, and J. B. Ketterson, J. Magn. Magn. Mater. 309, 317 (2007).

[41] F. G. Aliev, J. F. Sierra, A. A. Awad, G. N. Kakazei, D.-S. Han, S.-K. Kim, V. Metlushko, B. Ilic, and K. Y. Guslienko, Phys. Rev. B 79, 174433 (2009).

[42] A. Vansteenkiste, J. Leliaert, M. Dvornik, M. Helsen, F. GarciaSanchez, and B. Van Waeyenberge, AIP Adv. 4, 107133 (2014).

[43] N. A. Usov and S. E. Peschany, J. Magn. Magn. Mater. 118, L290 (1993).

[44] J. Ding, G. N. Kakazei, X. Liu, K. Y. Guslienko, and A. O. Adeyeye, Sci. Rep. 4, 4796 (2014).

[45] M. Noske, H. Stoll, M. Fähnle, A. Gangwar, G. Woltersdorf, A. Slavin, M. Weigand, G. Dieterle, J. Förster, C. H. Back, and G. Schütz, Phys. Rev. Lett. 117, 037208 (2016).

[46] K. Schultheiss, R. Verba, F. Wehrmann, K. Wagner, L. Körber, T. Hula, T. Hache, A. Kakay, A. A. Awad, V. Tiberkevich, A. N. Slavin, J. Fassbender, and H. Schultheiss, arXiv:1806.03910.

[47] M. J. Martínez-Pérez and D. Zueco, arXiv:1807.04075.

[48] I. Proskurin, A. S. Ovchinnikov, J. Kishine, and R. L. Stamps, arXiv:1809.10091.

[49] J. H. Han, Skyrmions in Condensed Matter (Springer, Berlin, 2017).

[50] J. Heebner, R. Grover, and T. A. Ibrahim, Optical Microresonators (Springer, London, 2008), Vol. 138.

[51] K. Y. Guslienko, V. Novosad, Y. Otani, H. Shima, and K. Fukamichi, Phys. Rev. B 65, 024414 (2001). 